\title{
The influence of specialty training, experience, discussion and reflection on decision making in modern restorative treatment planning
}

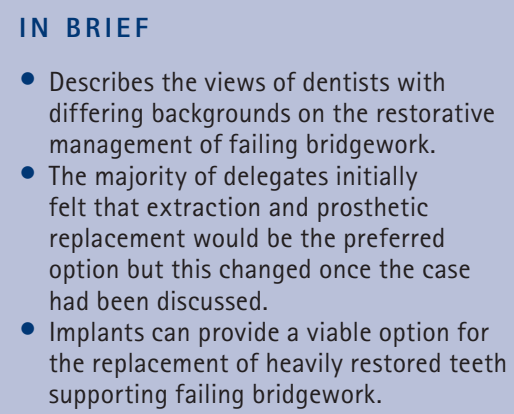

\author{
A. Alani, ${ }^{1}$ K. Bishop ${ }^{2}$ and S. Djemal ${ }^{3}$
}

Aims This study was designed to determine the effect of reflection and discussion of a group of dentists with differing backgrounds and qualifications in the management of failed endodontic treatment. Materials and methods During the Dental Pan-Society plenary session (16-17 November 2007) delegates $(n=393)$ were asked a series of questions on the management of a case with failed endodontic treatment of four maxillary incisors restored with linked crowns in a patient with a high smile line. The case had been previously posted on the conference website in addition to being presented on the day of the forum. Responses of delegates to predetermined questions and options on the management of the case were recorded using closed-circuit devices for each individual delegate. The questions were repeated after the case was opened up for discussion by the delegates in conjunction with a panel of leading experts. The discussion topics included the factors affecting the outcome of secondary root canal treatment, post-extraction changes and the options for prosthetic replacement including the provision of implants in the aesthetic zone. Results The initial response of the majority (58\%) of delegates favoured extraction and prosthetic rehabilitation over endodontic retreatment of the affected teeth. Following the discussion this figure reduced to $50 \%$. In respect to those individuals who were specialists, extraction was again the preferred option before the discussion for periodontists (74\%), prosthodontists (64\%) and restorative dentists (65\%). This was in contrast to endodontists who preferred endodontic retreatment, with only $30 \%$ identifying extraction as the treatment of choice. Following the discussion, the number of periodontists and endodontists who favoured extraction reduced by $3 \%$ and $5 \%$ respectively, whereas the number of prosthodontists and restorative dentistry specialists who preferred extraction increased by $2 \%$ and $4 \%$ respectively. Conclusion Reflection and discussion can make individuals reconsider their initial treatment decisions. These personal skills may become more significant when planning treatment for complex restorative cases.

\section{INTRODUCTION}

Good clinical practice is based on the ability to process evidence-based knowledge, reflect on previous experiences and apply these to a clinical setting when making decisions for treatment. ${ }^{1}$ The decisionmaking process for the restoration or the replacement of teeth is complex. ${ }^{2}$ Attempts have been made to rationalise the process and strategies have been devised to guide less experienced clinicians. ${ }^{1,3-6}$

${ }^{1 *}$ Specialist Registrar in Restorative Dentistry, Department of Restorative Dentistry, Newcastle Dental Hospital, NE2 4AZ; ${ }^{2}$ Consultant in Restorative Dentistry, Department of Restorative Dentistry, Morriston Hospital, Swansea, SA6 6NL; ${ }^{3}$ Consultant in Restorative Dentistry, Department of Restorative Dentistry, Kings College Hospital, Denmark Hill, SE5 9RS

${ }^{*}$ Correspondence to: Mr Aws Alani

Email: awsalani@hotmail.com

Online article number E4

Refereed Paper - accepted 6 August 2010

DOI: 10.1038/sj.bdj.2011.92

${ }^{\circ}$ British Dental Journal 2011; 210: E4
Despite these attempts, difficulty persists, as illustrated in studies that have examined the treatment planning of individuals with differing clinical backgrounds and experiences. ${ }^{1,2,5,7-9}$ For example, McCaul and co-workers examined the decision-making process of a group of different clinicians including specialist endodontists, consultants and specialist trainees in restorative dentistry as well as general dental practitioners. ${ }^{1}$ The results from this study highlighted the wide variety of reasons used by clinicians when reaching a diagnosis and by individuals with different specialties and experience when making the decision whether or not to treat a tooth endodontically. Interestingly, the greatest inter-observer agreement in this study was between specialist endodontists.

The reasons why clinicians choose a surgical approach to endodontic retreatment have also been examined. ${ }^{9}$ In a retrospective study, el-Swiah and Walker found that the most common reasons for clinicians to choose an apicectomy as the treatment of choice was either the presence of a post-core restoration or persistent problems such as suppuration or pain. ${ }^{10}$

With the success associated with dental implants and root canal retreatment it may become even more difficult to decide whether to endodontically retreat or extract a tooth. ${ }^{11-13}$ Although a number of systematic reviews have compared success rates of root canal treatment and survival of implant restorations, they have not been able to conclude which treatment option is the most appropriate. ${ }^{14-16}$ In addition, no study has attempted to evaluate how clinicians decide between either implants or to retain an endodontically compromised tooth and attempt retreatment.

The Dental Pan-Society conference is a triennial joint meeting between the 
specialist restorative societies in the UK (British Endodontic Society, British Society of Periodontology, British Society for Restorative Dentistry, and the British Society for the Study of Prosthetic Dentistry). Its aim is to facilitate discussion and debate on current issues in restorative dentistry with the first meeting held in Birmingham, UK in 2007. The societies represent both specialists and non-specialists and the conference is also open to non-members of the societies.

As part of the conference, a discussion forum centred around two clinical cases was included in the programme. Delegates were asked a series of questions where both endodontic retreatment and extraction and prosthetic replacement were viable options. In addition, recognised experts in the various restorative specialities contributed to the discussion.

The aim of this study was to assess the effect of reflection and discussion by delegates from different backgrounds in the decision-making process for one of these cases.

\section{MATERIALS AND METHODS}

Two complex restorative case studies were posted on the Pan-Society website (http:// www.pandental2007.org/case_studies. html) one month before the conference to allow delegates to familiarise themselves with the cases that were to be discussed (Figs 1-3). The results of one of these cases will be illustrated in this paper.

At the start of the session, the chair asked delegates a series of questions through a PowerPoint presentation (Microsoft, Virginia, USA) related to the experience and background of the delegates present. These included year of qualification, location of clinical practice, specialist status and the amount of NHS and private practice carried out (Figs 4-8). The responses to these and all other subsequent questions were recorded through a closed circuit recording system (Turning Technologies, Ohio, USA). Individuals were given a 30-second time interval to record their replies and delegates were encouraged to answer all questions. Unfortunately, a few failed to respond consistently, and were omitted from the study.

The case was illustrated and a series of predetermined questions relating to the potential management were presented to the audience, in particular whether endodontic retreatment or extraction and

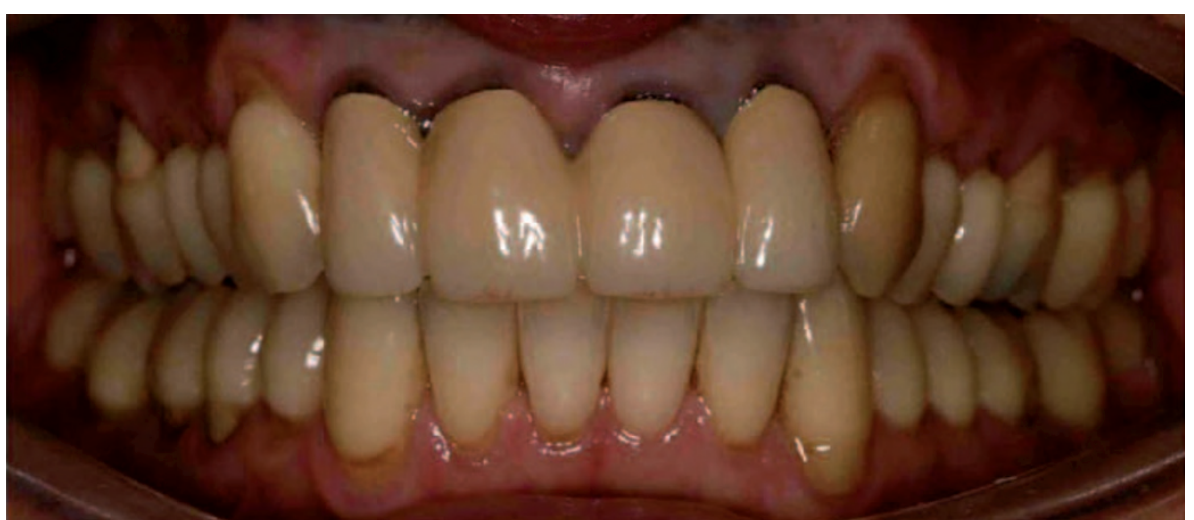

Fig. 1 Anterior linked crowns with sub-optimal margins. The patient had a high smile line and was concerned with anterior aesthetics

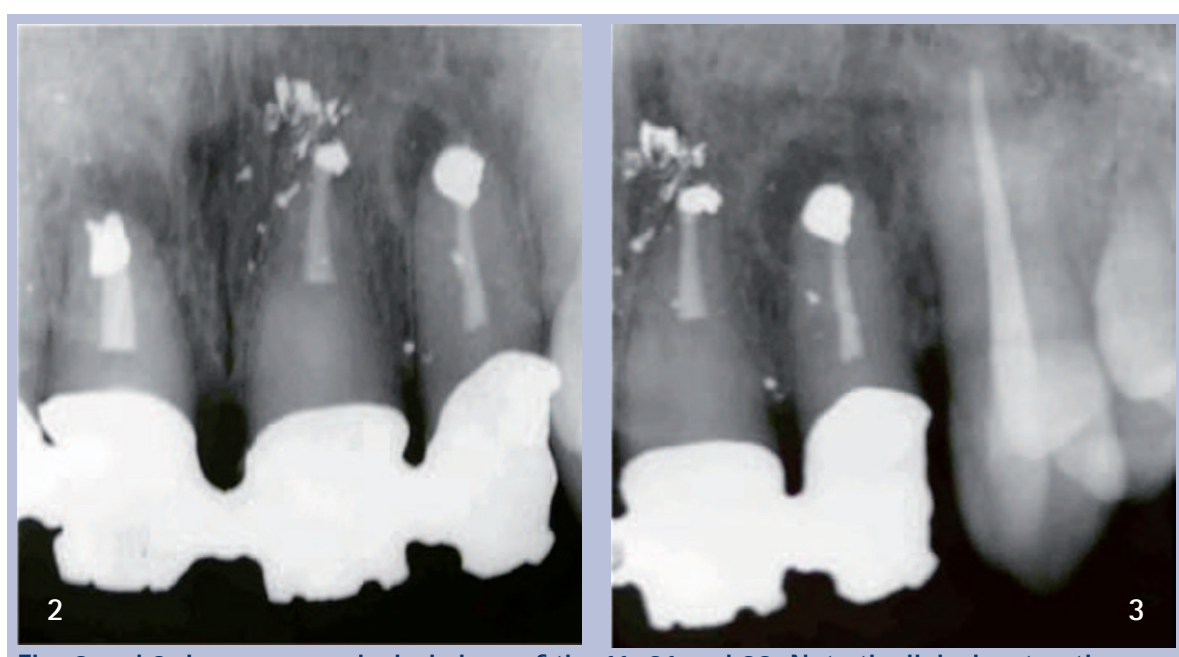

Figs 2 and 3 Long cone periapical views of the 11, 21 and 22. Note the linked restorations, the presence of previous orthograde and retrograde root canal treatments and persistent periapical lesions

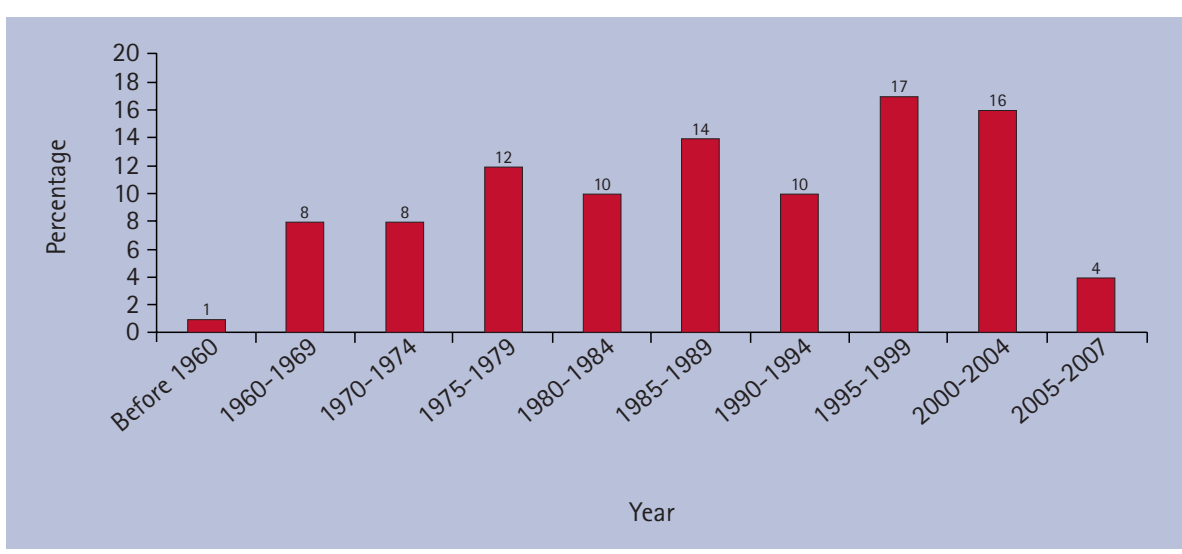

Fig. 4 Bar graph illustrating the year of qualification of delegates

prosthetic replacement would be the treatment of choice (Figs 9-12). The case was then discussed by a panel of four internationally recognised experts in the fields of prosthodontics, periodontics, endodontics and implantology. The discussion points were predetermined and included the issue of placing implants in the aesthetic zone, the question of soft tissue and bone quality post-extraction, the outcome of root canal retreatment and the restorability of endodontically treated teeth. At the end of the discussion, the delegates were asked question 1 again to see how they felt the case should be managed in light of the discussion.

\section{RESULTS}

Three hundred and ninety-three delegates took part in the survey, 252 


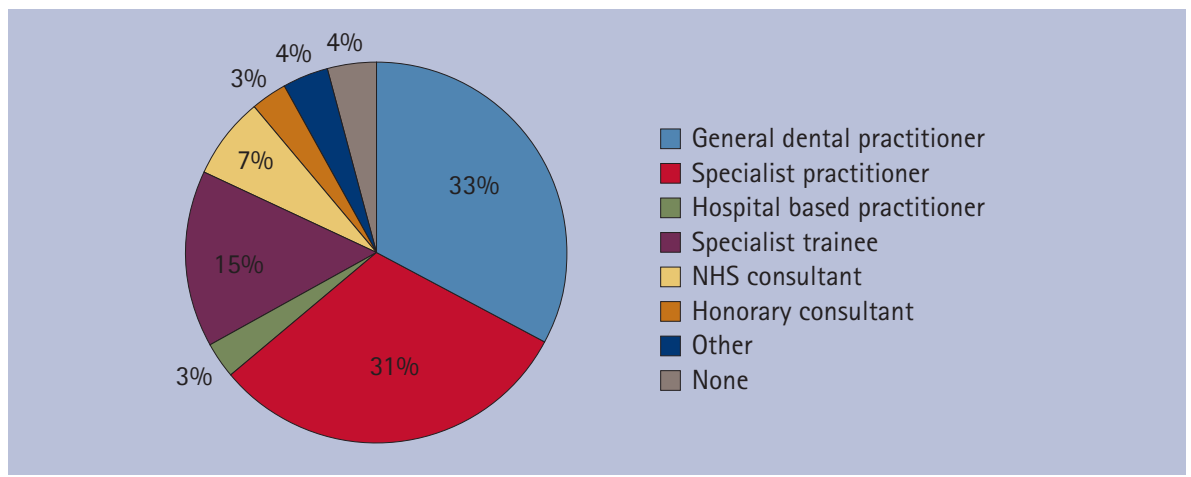

Fig. 5 Pie chart illustrating clinical practice of delegates

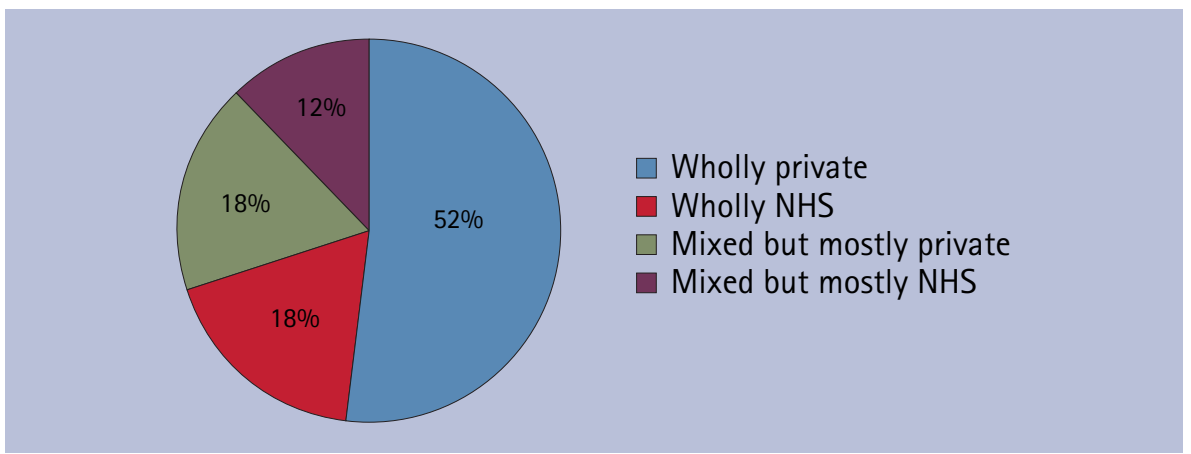

Fig. 6 Pie chart illustrating the clinical activity of delegates

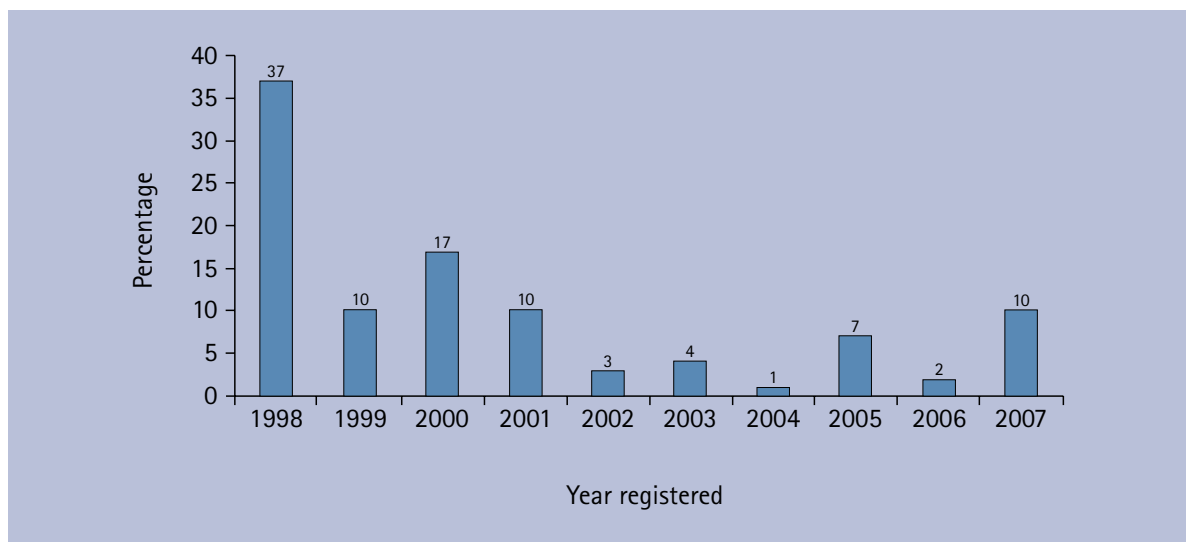

Fig. 7 Bar graph illustrating year registered as specialist

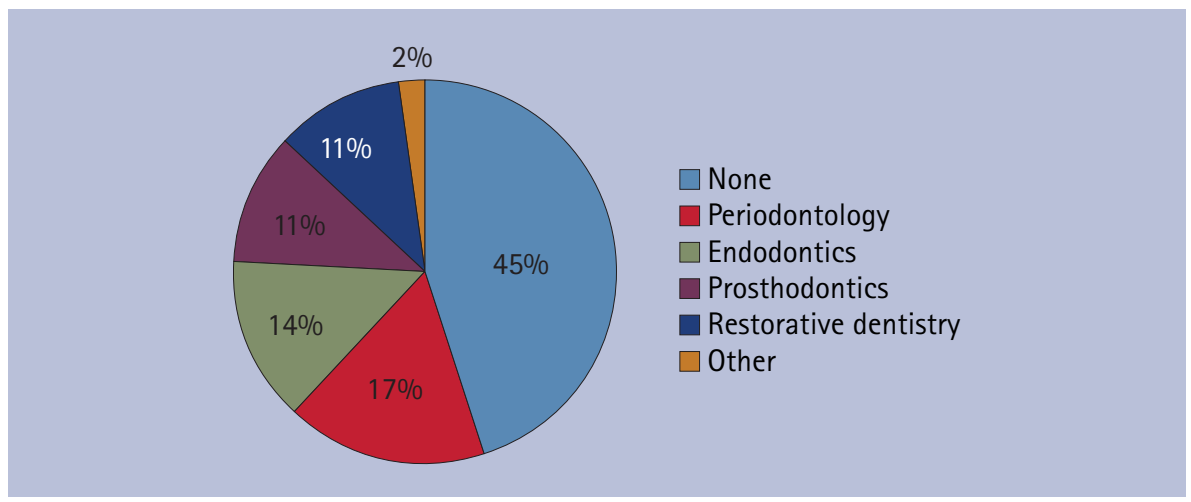

Fig. 8 Composition of specialist delegates

(64\%) of whom were male and 141 $(36 \%)$ female. The majority of delegates qualified after 1985 (Fig. 4) with 47\% (185) qualifying between 1990 and 2007.
Sixty-four percent of respondents (250) were general dental practitioners and high street specialists working in primary care with 28\% (110) being hospital-based staff of various grades (Fig. 5).
Fifty-two percent (205) of delegates worked solely within private practice, 18\% (70) wholly in the NHS and the remainder a mixture (Fig. 6). Of the delegates who were specialists (55\%, 216), 37\% (80) were first registered as such in 1998 (Fig. 7).

Seventeen percent of the delegates were specialist periodontists (66), 14\% specialist endodontists (55), 11\% specialist prosthodontists (43) and 11\% specialists in restorative dentistry (43). Two percent (eight) were specialists in areas 'other' than those above (Fig. 8).

\section{Response to specific questions}

Question 1: What are your initial thoughts on how this case should be managed?

When first asked this question, the majority of the delegates (specialists and non-specialists) felt that extraction and prosthetic replacement of the anterior teeth was the treatment of choice (58\%, 228). This included specialists in the 'other' category, 60\% (five) of whom favoured extraction and prosthetic replacement. The only exception was specialists in endodontics with the majority $(70 \%, 39)$ favouring endodontic retreatment (Figs 9-13).

The results also showed that all specialists regardless of the year registered chose extraction and prosthetic replacement as the preferred treatment option $(58 \%, 228)$.

The majority of specialist trainees (63\%, $36)$ and NHS consultants $(60 \%, 17)$ also considered extraction and prosthetic replacement the treatment of choice.

\section{Question 2: If you did attempt to retain the teeth what endodontic treatment would you initially plan to provide?}

In response to this question, 73\% (287) of all delegates (Fig. 14) felt that a combined orthograde and retrograde endodontic treatment would be required.

The year of qualification appeared to have an effect, with $81 \%$ (64) of the most recently qualified delegates (2000-2007) felt that both surgical and orthograde endodontics should be embarked upon while 71\% (223) of individuals qualifying between 1960-1999 felt that this was the most appropriate approach.

Specialism also appeared to have an effect, with general dental practitioners 
more in favour of a combined approach $(79 \%, 104)$ than primary care specialist practitioners (70\%, 85). Thirty percent (37) of these primary care specialist practitioners advocated attempting orthograde root canal treatment in the first instance.

Both NHS and honorary consultants showed similar results, with 33\% (13) considering conventional root canal retreatment and 66\% (26) favouring a combined approach.

Specialists in restorative dentistry were less in favour of a combined approach $(60 \%, 26)$ than other specialist or nonspecialist groups $(75 \%, 130)$. In addition, the proportion of restorative specialists who preferred orthograde endodontics as the sole method of treatment was greater than in other groups including endodontic specialists $(37 \%, 16)$.

Interestingly, of all the specialist delegates, only a small number of periodontists (4.5\%, three) considered a surgical-only approach.

Those delegates in wholly NHS practice were less likely to consider a combined approach $(66 \%, 47)$ compared to wholly private practitioners $(74 \%, 151)$, mixed but mostly private practitioners $(75 \%, 53)$ and mixed but mostly NHS practitioners $(73 \%, 34)$.

\section{Question 3: If endodontic retreatment proves to be successful what would you use to finally restore the teeth?}

Ninety-nine percent of the delegates felt that the teeth should be restored as single units rather than linked crowns. Of these 55\% (216) felt that the restoration should involve individual metal ceramic crowns while 28\% (111) considered individual highly sintered ceramic crowns the preferred option (Fig. 15).

Both hospital-based specialists and NHS consultants were more in favour of individual metal ceramic restorations (71\%, eight and 74\%, 20 respectively) in comparison to those individuals working in general or specialist practice in primary care $(55 \%, 138)$. Thirty percent (75) of the latter favoured highly sintered ceramic crowns and 15\% (38) favoured conventional all-ceramic crowns compared to 15\% (six) and 12\% (five) of hospital-based staff respectively.

The majority of specialists in restorative dentistry $(70 \%, 30)$ felt that metal ceramic

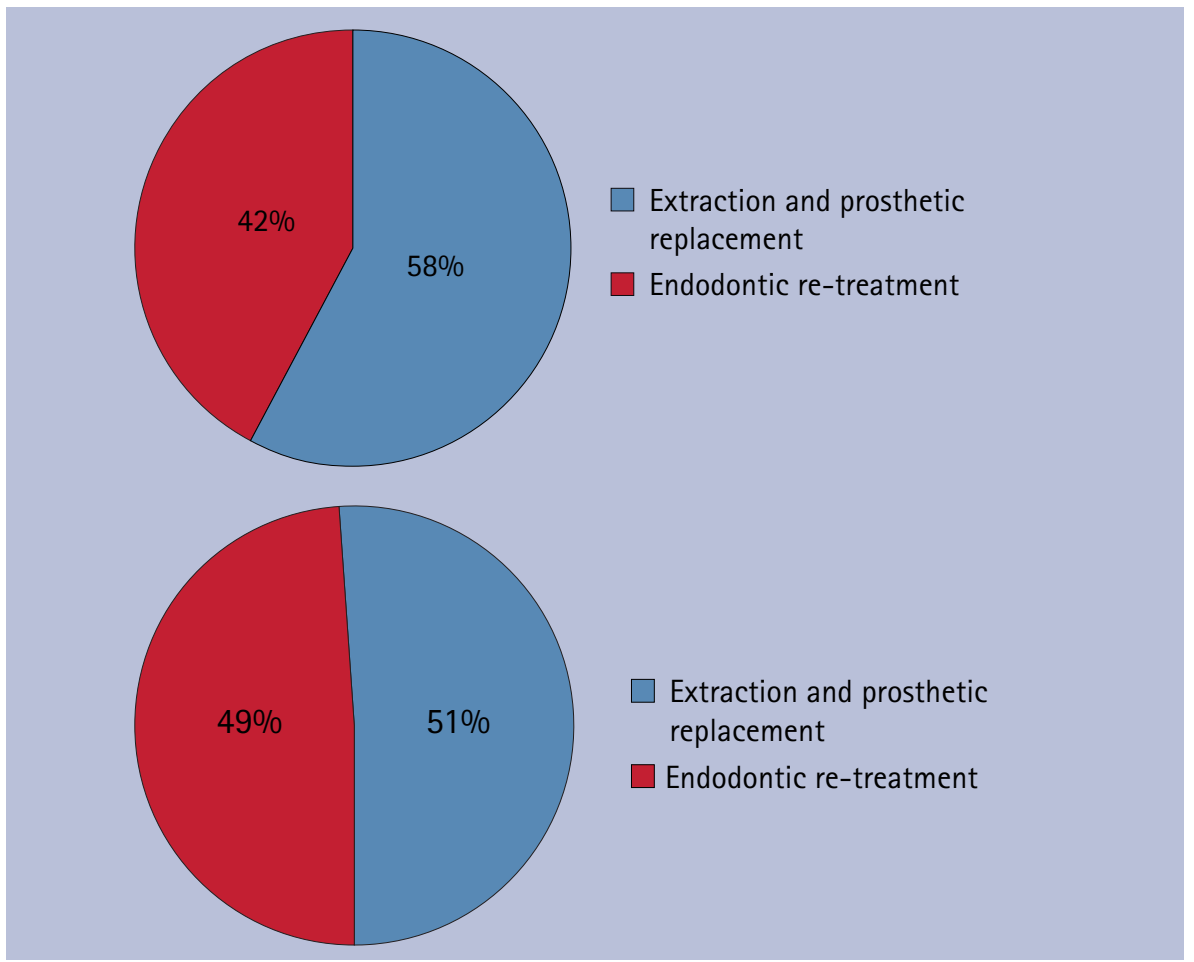

Figs 9 and 10 Pie charts illustrating pre- and post-discussion split of delegates on treatment planning decision for the case

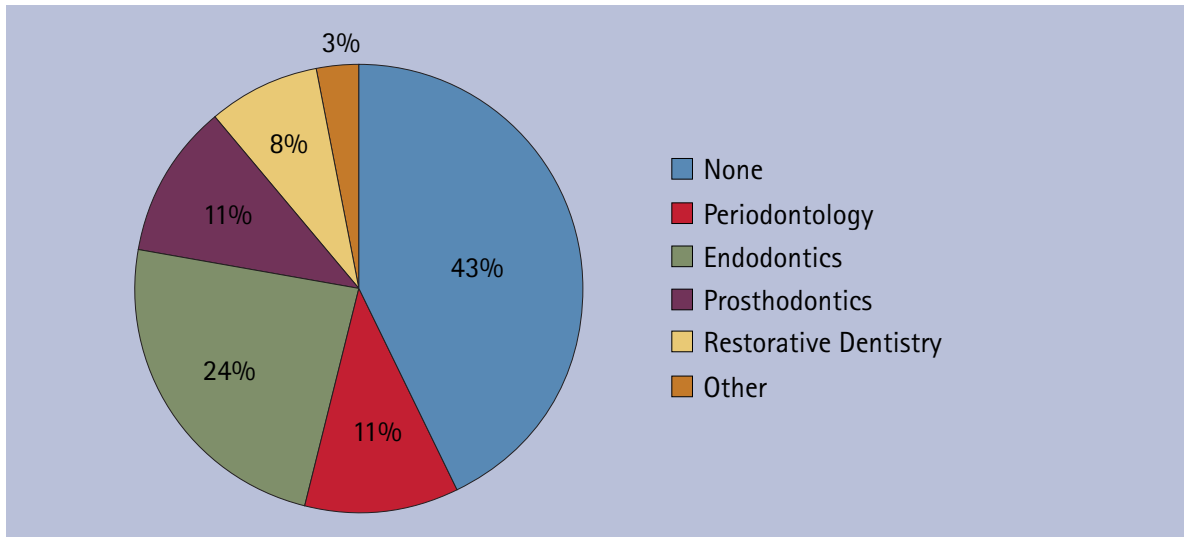

Fig. 11 Pie chart illustrating the specialist composition of delegates who initially considered endodontic retreatment

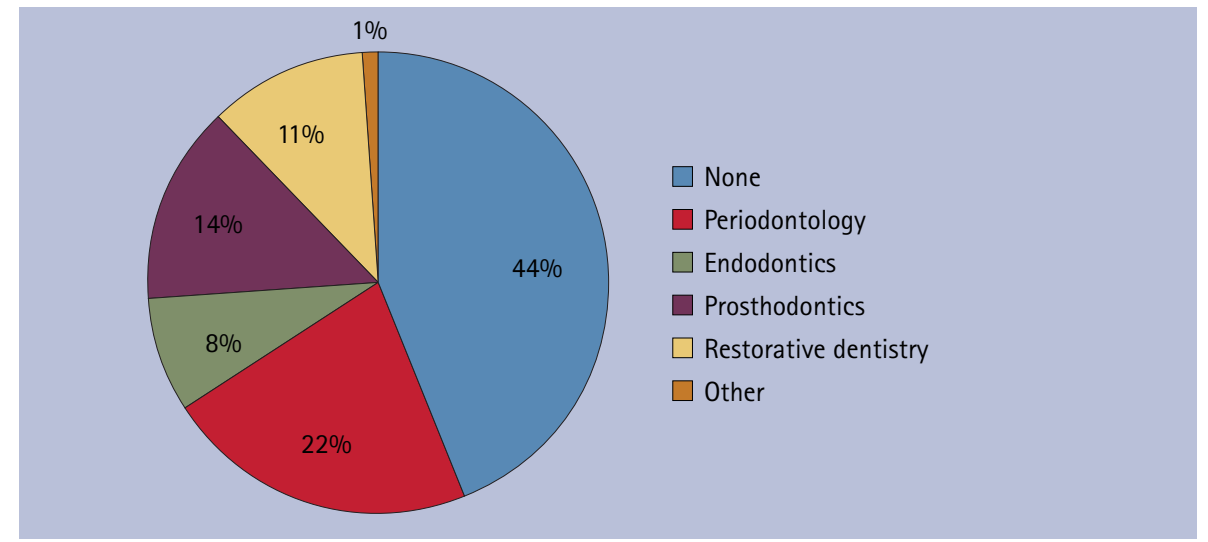

Fig. 12 Pie chart illustrating the specialist composition of delegates who initially considered extractions and prosthetic replacement

restorations were the restoration of choice in comparison to specialists in periodontics
(52\%, 35), endodontics $(61 \%, 33)$ and prosthodontics $(65 \%, 28)$. 
Question 4: If the teeth are extracted what do you think the definitive prosthesis should be?

The use of an implant-retained prosthesis was the most popular option for rehabilitation by all delegates $(68 \%, 267)$ and by all specialist groups. The next most popular was adhesive bridgework $(16 \%, 63)$ (Figs 16 and 17).

This result was also reflected by location of practice, with 82\% (100) of high street specialists and 75\% (44) of specialist trainees favouring this option (Fig. 18) and 70\% (19) of NHS consultants. In contrast, only 33\% (four) of honorary consultants were in favour of this option (Fig. 18).

Those delegates qualifying between 2000 and 2007 were less likely to consider implant rehabilitation than those delegates who qualified before this (Fig. 19). Interestingly, delegates who qualified between 2000-2007 were more likely to consider adhesive bridgework as an option $(21 \%, 17)$ than any of the other cohorts and especially those qualifying between 1970 and 1999 (14\%, 39).

Specialist practitioners were most in favour of implant rehabilitation (83\%, $100)$, followed by specialist trainees (76\%, 45), NHS consultants $(70 \%, 20)$, hospital based specialists (67\%, eight), and lastly general dental practitioners (59\%, 72). Specialist practitioners were least in favour of adhesive bridgework (7\%, nine) in comparison to the other groups $(24 \%, 62)$.

The option of removable partial dentures was generally not popular, with only $10 \%$ (six) of specialist trainees and 6\% (two) of NHS consultants choosing this treatment modality. It was interesting to note that none of the specialists in prosthodontics considered a removable partial denture as an option.

Specialists in restorative dentistry were most in favour of implant-based rehabilitation $(83 \%, 36)$, followed by prosthodontists, periodontists (both 82\%) and endodontists $(74 \%, 41)$. Conventional bridgework was not a very popular treatment strategy in this case as no restorative specialists, two endodontists (4\%), three periodontists (5\%) and three prosthodontists (7\%) chose this treatment modality (Fig. 17).

All specialists registered between 2005 and 2007 considered implant bridgework the treatment of choice. This was

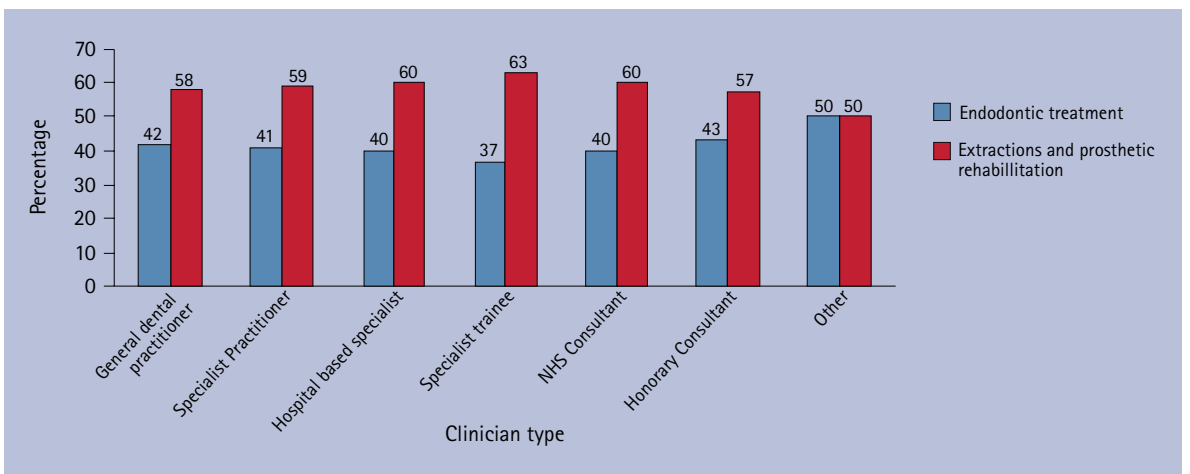

Fig. 13 Initial treatment planning decisions by clinician type

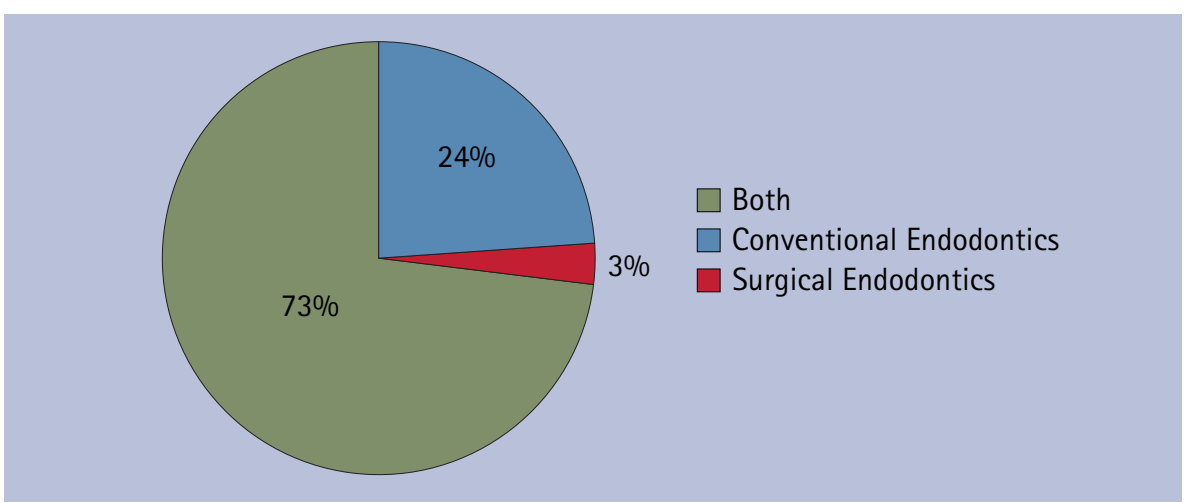

Fig. 14 Pie chart illustrating the delegates' thoughts on endodontic strategy

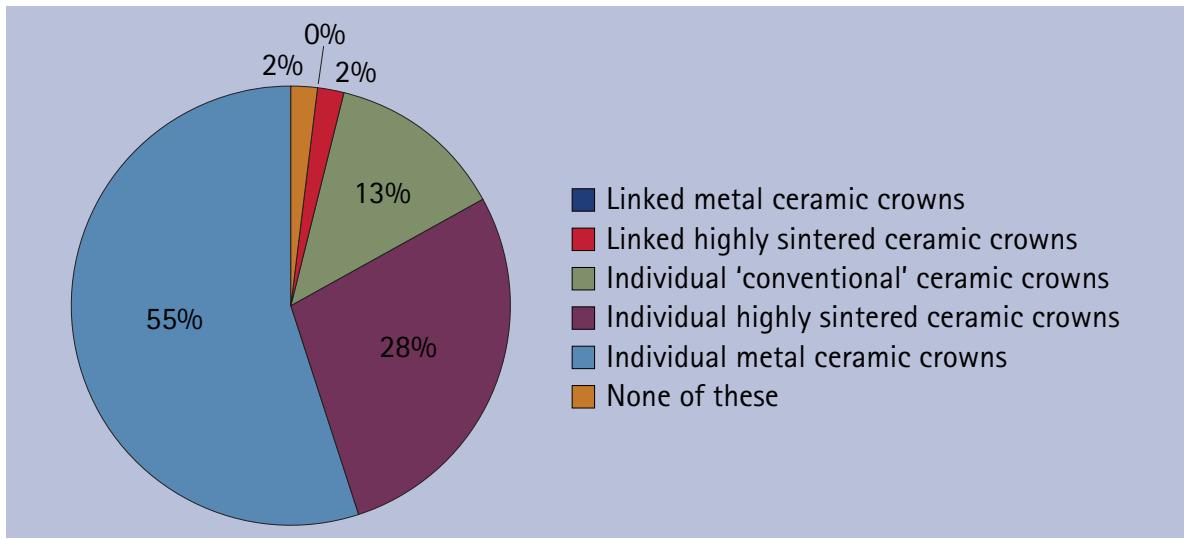

Fig. 15 Pie chart illustrating delegates' thoughts on definitive post-endodontic restoration

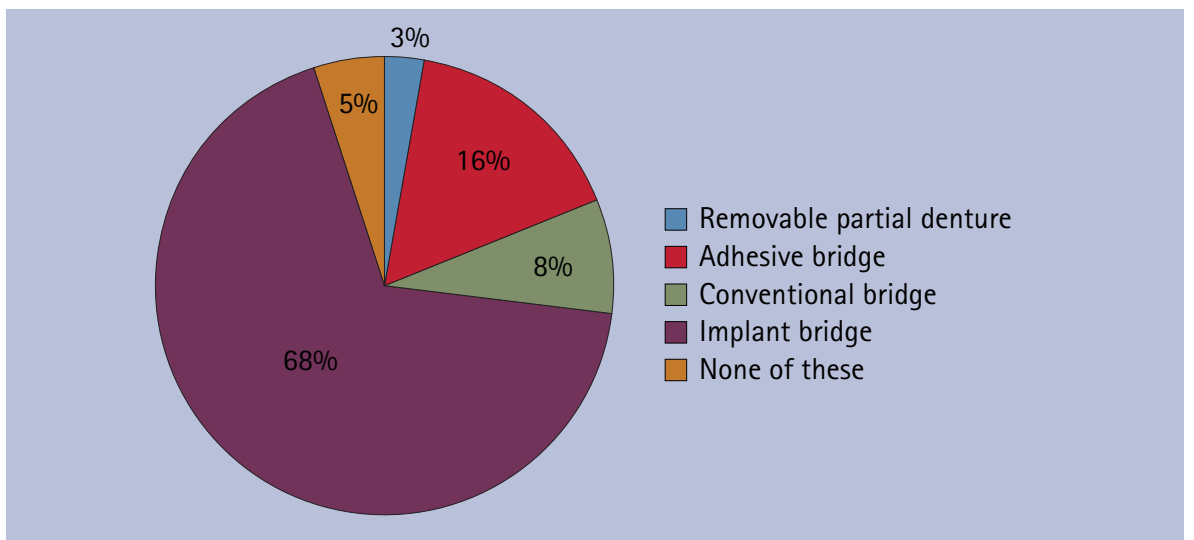

Fig. 16 Pie chart illustrating delegates' thoughts on definitive post-extraction restoration

greater than those registered in preceding years and especially in 1998 (58\%, 46). Individuals registered in 1998 were more likely to consider a removable partial denture (5\%, seven) or adhesive bridgework $(21 \%, 30)$ than individuals registered 
between 1999 and 2007 (0\% in favour of removable partial denture and 13\%, 18 in favour of adhesive bridgework).

Six percent of practitioners working in a wholly NHS environment considered a removable partial denture as an option in comparison to only $1 \%$ (two) of wholly private practitioners. NHS practitioners were less likely to consider implant bridgework $(64 \%, 45)$ than those working in the private sector $(76 \%, 155)$.

\section{DISCUSSION}

The presented results illustrate the different treatment decisions by a group of dentists from a variety of backgrounds and with different levels of experience and qualifications. Although there was representation from all of the demographic categories identified at the design stage of the study, there was no way of ensuring that this was a representative cohort of UK dentists. As a result there is no way of ensuring that results were completely representative at the time of data recording.

The demography of individuals attending such a large-scale meeting incorporating four specialist societies was varied. More than a third (37\%) of individuals had been qualified less than ten years at the time of the conference. The majority (57\%) of specialists qualified between 1980 and 1999. This could reflect the increased awareness of the need for continuing professional development for more recently qualified dentists as well as the need to gain more knowledge and understanding. The new prospect of revalidation every five years for specialists may also have been an additional factor. The meeting itself was organised incorporating parallel sessions for individual societies culminating in the plenary session on the final day. This allowed for a variety of subject matter to be available for delegates which may have attracted younger nonspecialist clinicians still considering where their future specialist interest may lie.

\section{Effect of year of qualification}

A greater proportion of the most recently qualified delegates was more likely to consider orthograde and retrograde endodontics than counterparts qualifying in preceding years. They may have not considered the complications of performing both these procedures, that is, further shortening of already apicected roots,

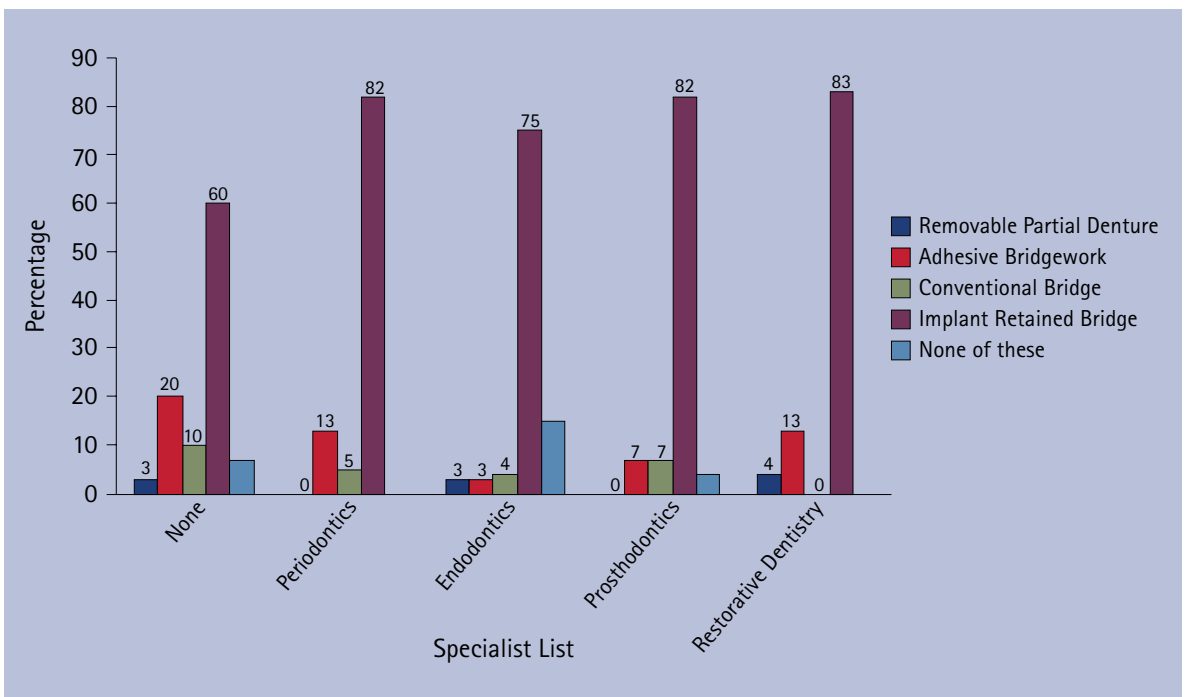

Fig. 17 Choice of definitive restoration by specialty

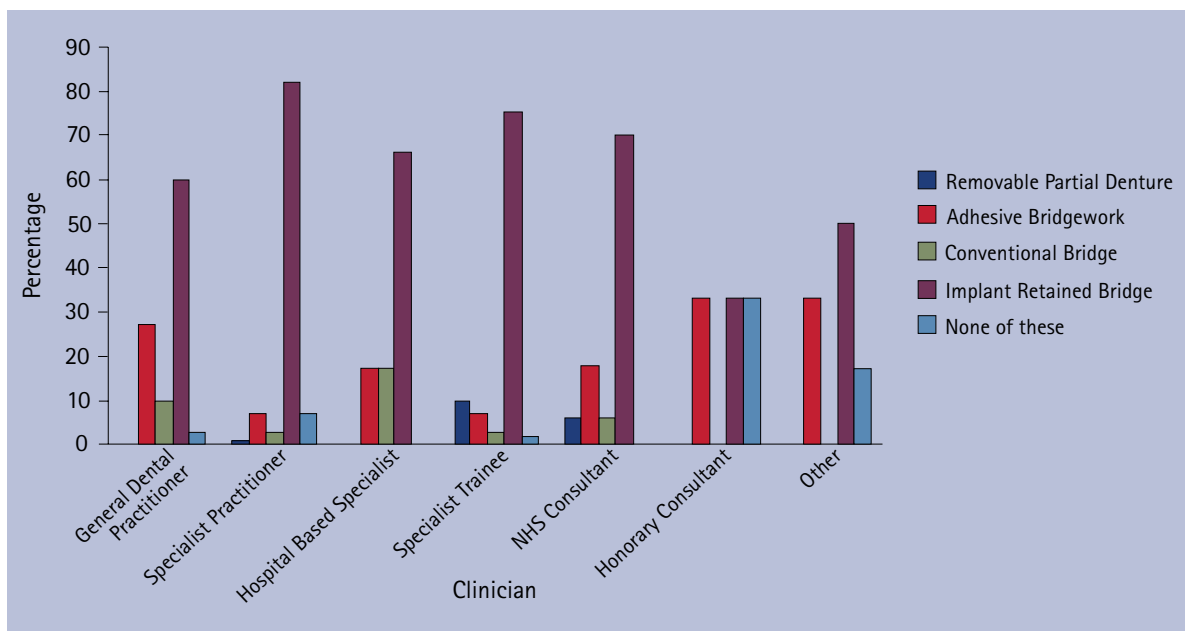

Fig. 18 Bar graph illustrating choice of definite restoration and clinician type

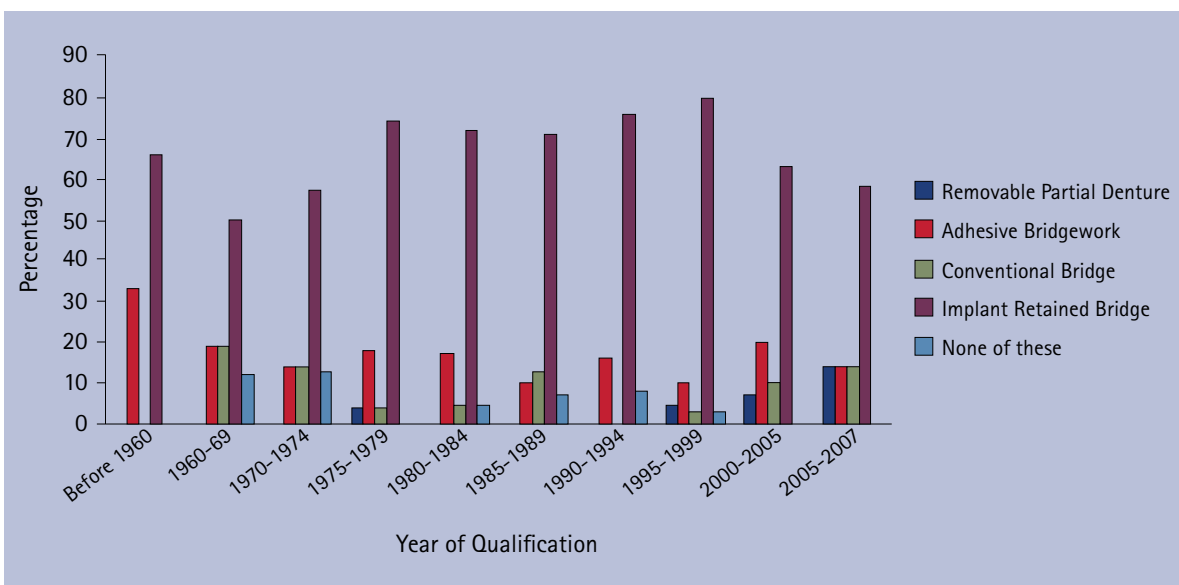

Fig. 19 Bar graphs illustrating the year of qualification of delegates and choice of definitive restoration if the teeth were to be extracted

further compromising the limited amount of supragingival tissue, another surgical episode that would compromise amount of bone and soft tissue for subsequent implant placement. This may illustrate the limited experience this cohort has in dealing with complex restorative problems.
A smaller proportion of most recently qualified delegates were less likely to consider implant-based rehabilitation than those qualified in preceding years. This may signify a lack of experience in providing implant-based rehabilitation by this cohort. Interestingly, this 
cohort was more likely to consider adhesive bridgework than previous cohorts. Delegates who had been qualified for longer were less in favour of the adhesive option. This may be related to their previous experiences of adhesive failures. Current research has resulted in a better understanding of the adhesive process and techniques in improving long-term success rates may have been more familiar to recently qualified delegates and specialist trainees. ${ }^{17}$

Delegates qualifying recently were more likely to reconsider their decisions postdiscussion. This may represent a lack of knowledge and realisation of the potential pitfalls of the case and possibly the inability to deal with these. In contrast, these large changes in opinion may be the result of the discussion process and show reflection by this cohort with an open-minded attitude towards treatment planning.

\section{Effect of year of specialist registration}

Of the specialists that were present at the conference, more than a third (37\%) had been first registered as such in the year of inception of the specialist lists in 1998. The majority of these individuals are likely to have been 'grandfathered' onto their respective specialist lists but would include individuals who had undertaken structured training programmes and some who had not but had demonstrated equivalence. They also include the most experienced of the specialist groups.

There was a consensus among all specialists on their initial thoughts that the case should be managed by extraction and prosthetic replacement. The treatment of choice, however, if the teeth were extracted, differed between the different cohorts. The most recently registered specialists (2005-2007) were all in favour of implant-based rehabilitation, in contrast to individuals registering earlier but especially in 1998 (58\%). Individuals registering in 1998 may have considered implant-based rehabilitation as the treatment of choice but may not have chosen this because of their experience and confidence in their skills in providing a successful result with the other options presented. This was apparent where 5\% of the 1998 cohort considered a removable partial denture and 21\% considered adhesive bridgework, in comparison to $0 \%$ and 13\% respectively of those registered between 1999 and 2007.

Post-discussion changes of how the case should be managed varied between the 1998 cohort and the remaining specialists. The 1998 cohort became less in favour of extractions while those registering later became more in favour. As the 1998 cohort is more experienced, the discussion of problems associated with either implant rehabilitation or endodontic retreatment may have triggered reconsideration of their initial choices.

\section{Effect of type of specialist}

The majority of specialists felt that the case should be managed by extraction and prosthetic replacement. The exception to this were specialist endodontists. This is not surprising taking into account this cohort's skills in dealing with failed primary root canal treatment and their surgical skills in retrograde management.

If the endodontic approach was to be embarked upon, specialists in restorative dentistry were less in favour of a combined non-surgical and surgical approach than other specialists or non-specialist groups. A possible explanation may be that teeth that have a history of previous apical surgery will already have reduced root length and if the teeth presented were to be apicected for a second time then the remaining residual root length may render the tooth severely compromised. Another consideration by this cohort may have been the repeated surgical interference in this site. Flap reflection for a second surgical episode may have further compromised soft tissue health and bone mass if subsequent implant therapy was required. The pragmatic approach of simple orthograde root canal treatment in the first instance may have been chosen by some individuals with these factors in mind.

Once endodontics was considered a success, the modes of definitive restorations varied between different specialist groups. Restorative dentistry specialists (70\%) were more in favour of individual metal ceramic restorations than specialists in periodontology (52\%), endodontics (61\%) and prosthodontics (65\%).

Hospital-based specialists and NHS consultants (72\%) were more in favour of metal ceramic restorations than those working in specialist and general practice (55\%). This may reflect the different patient cohorts seen by the two groups. There may be a greater provision of cosmetic restorations in primary care practices in comparison to hospital-based provision. The decision to restore using all-ceramic restorations as opposed to metal-ceramic combinations may provide a better aesthetic result where previous metal-ceramic restoration can become unsightly around the margin in a patient with a high smile line (Fig. 1).

Interestingly, when considering definitive treatment post extraction, the removable partial denture option was not chosen among any of the prosthodontist's present. This is surprising considering the skill set and knowledge of the cohort. It is possibly explained by the greater emphasis on providing fixed options which may have higher social acceptability.

Specialists in restorative dentistry were most in favour of the implant-based option in comparison to the other specialist groups. This may be in part due to their exposure to all three specialties during their training and so provide an overarching viewpoint on how the case should be managed where implants would be the ideal coupled with confidence in their delivery.

Conventional bridgework was less popular than implant based and adhesive options especially with specialists in endodontics and restorative dentistry and is likely to be due to knowledge of the effect of conventional preparations on otherwise intact teeth. Endodontic complications of conventional preparations are well documented and may have influenced this decision. ${ }^{18-20}$

Post discussion opinions among the specialists changed, some becoming more in favour of extraction and prosthetic replacement (restorative dentistry and prosthodontics) and others becoming less in favour (Fig. 20). The proportion of specialists in periodontology favouring extraction and prosthetic replacement reduced by $3 \%$. Similarly, the number of endodontists favouring extraction and prosthetic replacement also reduced. These results may reflect the multidisciplinary nature of training in restorative dentistry in the single specialties and in particular the ability to plan treatment for a multispecialty case. 


\section{Effect of location of practice}

Seventy-nine percent of general dental practitioners were in favour of a combined approach to root canal treatment in the first instance compared to specialists working in primary care (68\%) and consultants in secondary care (66\%). This may be considered atypical as the treatment of the case using surgical intervention may be more suited to a specialist skill set, although this may also signify knowledge and experience of the difficulties that a combined approach may present with.

High street specialists and specialist trainees (82\%) were in favour of implantbased rehabilitation if the teeth were to be extracted. This may be due in part to the environment that delegates were currently working in, where the demand for implant restorations maybe high. The proportion of NHS consultants was slightly less (70\%). This may represent a financial constraint on this cohort to provide implants within strict selective criteria in secondary care despite dealing with high priority groups. It may also signify a proportion of this cohort having the confidence to provide restorations not involving the need for osseointegration.

General dental practitioners were comparatively the least in favour of implant based rehabilitation (59\%). Although the majority were in favour of implants the likelihood that this is provided on a private basis is high and there is as yet no NHS remuneration for implant therapy within the general dental services contract. ${ }^{21,22}$

Specialist practitioners were least in favour (7\%) of adhesive bridgework in comparison to all other groups (24\%). This may be associated with concerns about the predictability of such bridgework especially when providing restorations in a private environment. As the bridgework would be spanning a wide space, specialist delegates may have perceived a risk of debond or failure to be fairly high. ${ }^{17}$

The option of removable partial dentures was not popular by any grades. This may be related to the greater social acceptability and demand for fixed restorations in comparison to a removable denture.

\section{Effect of practice}

As would be expected, clinicians working in the private sector (76\%) were more likely to consider implant bridgework than

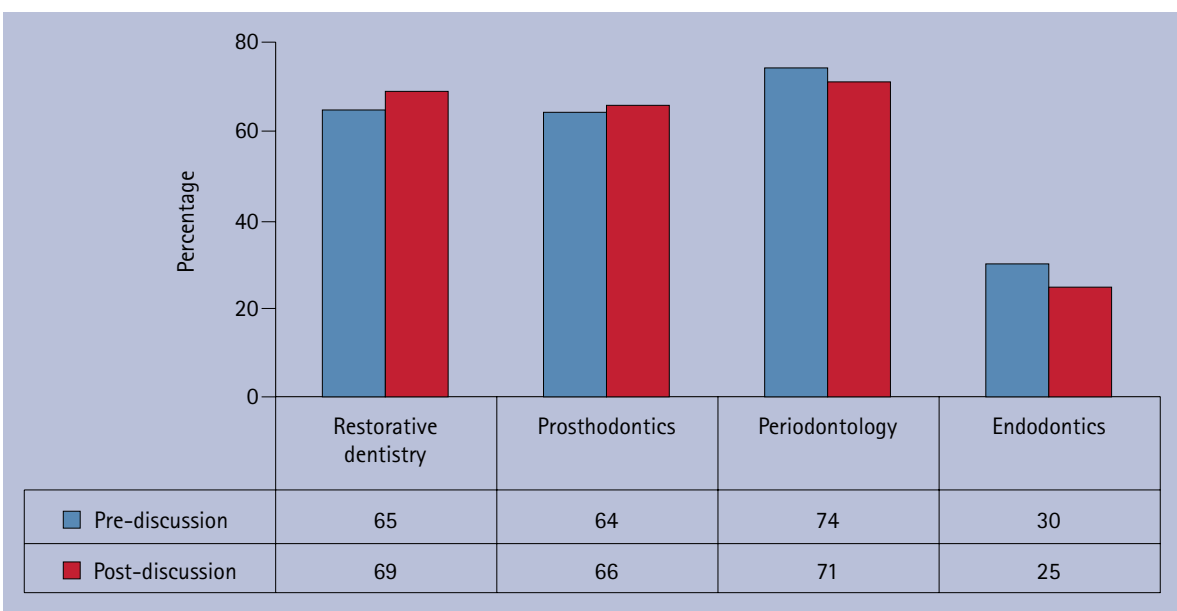

Fig. 20 Percentage of delegates (by specialty) in favour of extraction and prosthetic rehabilitation before and after discussion

those working in the NHS (64\%). This difference could be considered marginal when considering that the provision of implants in the NHS is likely to be confined to the secondary care sector with strict selection criteria.

\section{Effect of debate and reflection}

The process of discussing the case with experts from each of the restorative fields and time to reflect on the initial decision did cause some delegates to reconsider their initial thoughts. This was reassuring in that dental professionals were able to change their mind and not stick dogmatically to a chosen approach in light of new information provided by the expert panel.

Differences between different specialties in deciding whether to endodontically retreat or extract and prosthetically replace yielded interesting results both pre- and post-discussion. As would be expected, specialists in endodontics were strongly in favour of endodontic retreatment. Even for an endodontic specialist the surgical intervention would provide a significant challenge in removing a further $3 \mathrm{~mm}$ of apical tissue and rendering the teeth more compromised. Regardless of this the majority of endodontists were in favour of endodontic retreatment both initially (70\%) and post-discussion (75\%). This viewpoint was in contrast to the other specialist cohorts, the majority of which were in favour of implants pre- and postdiscussion (Fig. 16). The number of specialists in restorative dentistry favouring extraction increased after the discussion from $65 \%$ to $69 \%$. This may illustrate greater awareness of the difficulties of implant placement post-extraction but confidence in the management of these. In contrast the numbers of specialists in periodontology favouring extraction and prosthetic replacement reduced from $74 \%$ to $71 \%$. This may signify a greater awareness post-discussion of difficulties in managing the soft tissue post-implant placement in a patient with high aesthetic demands.

Our results show that most practitioners consider implants a viable option when faced with teeth of questionable endodontic and prosthodontic prognosis. This may signify a paradigm shift in the advent and popularity of osseointegrated restorations. Where previously heroic efforts may have been made to prevent the need for a removable restoration or long-spanning bridgework, implants now allow practitioners to provide fixed restorations following extractions. The initial responses of all delegates would confirm such thinking. 58\% of all delegates favoured extraction and prosthetic replacement in favour of endodontic retreatment. $68 \%$ of all delegates favoured implant-based rehabilitation if the teeth were extracted. The discussion of the case included the possible implications of post-extraction hard and soft tissue changes and how these would affect subsequent implant placement. The difficulty in managing the aesthetic zone in a patient with a high smile line was also discussed. The influence of both the discussion forum and the input from opinion leaders in the monospecialties was illustrated in the significant difference of opinion of the delegates when questioned a second time on definitive treatment of either endodontics or extraction. The 
delegates were split 50/50 with a subsequent post-discussion change towards endodontic re-treatment. Delegates may have reflected on their initial decisions, quick to discount the potential of tooth retention in addition to further consideration to the potential difficulties of implants in the aesthetic zone.

The variation between year of specialist registration and post-discussion changes was interesting. Those specialists registering in 1998 became less in favour of extractions (58\% to 51\%) while those remaining were more in favour, although this was only marginal (58\% to 60\%). This may signify differences in knowledge and experience of these cohorts, considering the majority of those registering between 1999 and 2007 were part of formalised training programmes in comparison to the 'grandfathered' cohort.

The proportion of delegates who reconsidered their initial decision was the greatest among those qualifying most recently. The percentage of delegates favouring extraction and prosthetic replacement in the 2000-2007 cohort changed from 57\% to $37 \%$ post-discussion. This may signify the limited amount of experience in treating what is a multifactorial case and not being aware of important issues such as restorability of compromised teeth, bone mass and soft tissue health for prospective implant placement. Older cohorts were less likely to reconsider their initial choice of either endodontic retreatment or extraction and prosthetic replacement; the greater experience of this cohort was likely to have contributed to this.

These post-discussion changes may signify a greater need for reflection and consideration of clinical decisions among dentists at all grades. Although implants may initially have seemed the obvious option for some delegates, this study illustrates the need for due consideration of all the factors involved in their provision. The knowledge of what is possible but may not be practical or predictable may only come with experience.

Anecdotal evidence and personal experience may influence clinical practice, and this coupled with expert opinion may determine our everyday treatment decisions, particularly when the evidence base is lacking. ${ }^{23}$

\section{CONCLUSION}

This study outlines the varied opinions of a group of delegates in the management of failing endodontic treatment. The study highlights the challenges in planning treatment for cases while considering newer treatment modalities such as implant-based rehabilitation.

Initially the majority of delegates felt that implant-based rehabilitation was the option of choice when faced with compromised tooth units. This opinion changed when the relative challenges of providing implant rehabilitation were highlighted by the expert panel and discussed among the delegates. This change could be attributed to reconsideration of initial judgements and the highlighting of issues that at first were not readily apparent.

The process of reflection and discussion can be seen as a valuable tool in everyday clinical practice. These skills may become more significant as the treatment options for the restoration or replacement of compromised teeth include advanced restorative procedures such as the placement of multiple implants in the aesthetic zone.

1. McCaul L K, McHugh S, Saunders W P. The influence of specialty training and experience on decision making in endodontic diagnosis and treatment planning. Int Endod J 2001; 34: 594-606.

2. Söderfeldt $B$, Palmqvist $S$, Eriksson $T$, Kronström $M$, Carlsson G E. A questionnaire instrument to assess clinical decision-making in prosthodontics among general practitioners. Acta Odontol Scand 1996; 54: 314-319.

3. Cosyn J, De Bruyn H. Interclinician disparity in periodontal decision making: need for consensus statements on surgical treatment. J Periodontal Res 2007; 42: 311-317.

4. Redford M, Gift H C. Dentist-patient interactions in treatment decision-making: a qualitative study. J Dent Educ 1997; 61: 16-21.

5. Reit C, Gröndahl H G. Endodontic decision-making under uncertainty: a decision analytic approach to management of periapical lesions in endodontically treated teeth. Endod Dent Traumatol 1987; 3 : 15-20.

6. Ríos Santos J V, Castelló Castañeda C, Bullón P. Development of a computer application to help in the decision-making process in teaching dentistry. Med Oral Patol Oral Cir Bucal 2008; 13: E65-70.

7. Ibbetson R J, Hemmings K W, Ward V J. Variations in planning fixed bridgework-a group of dentists at a case-based postgraduate course. Br Dent J 1999; 187: 159-163.

8. Pagonis T C, Fong C D, Hasselgren G. Retreatment decisions-a comparison between general practitioners and endodontic postgraduates. J Endod 2000; 26: 240-241.

9. Smith J W, Crisp J P, Torney D L. A survey: controversies in endodontic treatment and re-treatment. J Endod 1981; 7: 477-483

10. el-Swiah J M, Walker R T. Reasons for apicectomies. A retrospective study. Endod Dent Traumatol 1996; 12: 185-191.

11. Brånemark $\mathrm{PI}$, Adell $\mathrm{R}$, Breine $U$, Hansson $\mathrm{B} \mathrm{O}$, Lindstrom J, Ohlsson A. Intra-osseous anchorage of dental prostheses. I. Experimental studies. Scand J Plast Reconstr Surg 1969; 3: 81-100.

12. Brånemark $\mathrm{PI}$, Hansson $B \mathrm{O}$, Adell $\mathrm{R}$, Breine $U$, Lindstrom J, Hallén O. Osseointegrated implants in the treatment of the edentulous jaw. Experience from a 10-year period. Scand J Plast Reconstr Surg Supp/ 1977; 16: 1-132.

13. Ng Y L, Mann V, Gulabivala K. Outcome of secondary root canal treatment: a systematic review of the literature. Int Endod J 2008; 41: 1026-1046.

14. Torabinejad M, Anderson P, Bader J et al. Outcomes of root canal treatment and restoration, implantsupported single crowns, fixed partial dentures, and extraction without replacement: a systematic review. J Prosthet Dent 2007; 98: 285-311.

15. Iqbal M K, Kim S. For teeth requiring endodontic treatment, what are the differences in outcomes of restored endodontically treated teeth compared to implant-supported restorations? Int J Oral Maxillofac Implants 2007; 22 Suppl: 96-116.

16. Blicher B, Baker D, Lin J. Endosseous implants versus nonsurgical root canal therapy: a systematic review of the literature. Gen Dent 2008; 56: 576-580.

17. Djemal $S$, Setchell $D$, King P, Wickens J. Long-term survival characteristics of 832 resin-retained bridges and splints provided in a post-graduate teaching hospital between 1978 and 1993. J Oral Rehabil 1999; 26: 302-320.

18. Randow K, Glantz P O. On cantilever loading of vita and non-vital teeth. An experimental clinical study. Acta Odontol Scand 1986; 44: 271-277.

19. Cheung G S, Lai S C, Ng R P. Fate of vital pulps beneath a metal-ceramic crown or a bridge retainer. Int Endod J 2005; 38: 521-530.

20. Habsha E. The incidence of pulpal complications and loss of vitality subsequent to full crown restorations. Ont Dent 1998; 75: 19-21,24.

21. Vartoukian SR, Algraffee H. Does the referral and selection for NHS-funded dental implant treatment in the UK follow national guidelines? Ann R Coll Surg Eng/ 2007; 89: 247-251.

22. Field J C, Rousseau N, Thomason J M et al. Facilitation of implant provision in primary care. $\mathrm{Br}$ Dent J 2009; 207: E20.

23. Harwood C L. The evidence base for current practices in prosthodontics. Eur J Prosthodont Restor Dent 2008; 16: 24-34. 